\title{
BUILDING QUALITY BUILDERS: LESSONS LEARNED FROM A COMPANYWIDE TRAINING ON BEHAVIOR-BASED QUALITY
}

\author{
Paz Arroyo ${ }^{1}$ and Sulyn Gomez ${ }^{2}$
}

\begin{abstract}
This paper describes the process of designing, testing, and adjusting a virtual workshop called Building Quality Builders (BQB) to train a general contractor's employees on a Behavior-Based Quality (BBQ) approach with the purpose of increase implementation and reduce re-work. The paper summarizes a two-year journey that started in March 2019 and ramped up in 2020 due to the increased use of virtual training caused by Covid-19 pandemic. This paper describes the continuous improvement process and the lessons learned along the way. Lessons learned from developing and implementing this training are 1) BQB workshop main purpose to help participants improve the delivery of quality has been achieved by including a commitment to action from participants who took the workshop, 2) BQB format and content can be constantly improved if feedback from participants is being used for continuous improvement, and 3) BQB workshop is highly recommended by participants who took it, the recommendation extends to everyone in the company due to the benefits participants identified from BQB.
\end{abstract}

\section{KEYWORDS}

Behavior-based quality (BBQ), virtual workshops, quality.

\section{INTRODUCTION}

During 2019 - 2020, DPR Quality Group developed a virtual workshop called Building Quality Builders (BQB) to lead DPR teams through activities to prepare them with skills and resources to align and strategize on their quality implementation plan. The workshop was developed with the main intent of helping participants to create an action plan for the team to implement DPR's Behavior-Based Quality (BBQ) approach (Spencley et al. 2018, Gomez et al. 2019, and Gomez et al. 2020). The workshop helps participants to identify Distinguishing Features (DF) from all stakeholders' perspectives, to manage DF's timely, to agree on Measurable Acceptance Criteria (MAC) before the scope of work is handed off, and to communicate MAC to the field to have clarity on the work to do and ultimately avoid surprises (e.g., defects, rework, owner's dissatisfaction). The workshop provides tools that teams need to be more successful at identifying risks and be more proactive in the alignment of stakeholders' expectations to meet stakeholders' expectations.

Quality Leader, DPR, San Francisco, CA, PazA@dpr.com, orcid.org/0000-0002-8098-8172

$\mathrm{PhD}$ Candidate, Civil and Environmental Engineering Department, University of California, Berkeley, CA 94720-1712, USA, sulyn@ berkeley.edu, orcid.org/0000-0003-2367-9880 
The paper summarizes a two-year journey that started in March 2019, and it accelerated in 2020 given DPR's corporate decisions to deal with Covid -19 pandemic challenges. This workshop responded to 1 ) the need to improve quality management at the project level, 2) avoid rework and 3) an increased acceptance for virtual workshops. During Covid-19, with many teams working remotely, a larger number of teams were willing to join the workshop virtually. Up to date, 253 have taken the workshop in a total of 26 rounds. Participant's roles vary, including superintendents, project managers (PMs), and project engineers (PEs) including teams in all US Regions, Europe, and India.

Throughout conducting the workshops, we learned:

1. How to engage diverse teams,

2. how to change the quality mindset from quality is something that happens after I do the work towards a proactive behavioral approach for quality, and

3. how to foster actions for implementing what was learned after taking the workshop.

For developing this workshop, we identified some of the behaviors that we want teams to display and created tools to support teams when having to lead difficult conversations to align expectations with owners, architects, and trade partners.

In this paper, we will share our journey to create the workshop, our struggles, the changes we implemented, and the results. We believe this process is not only useful for practical resons, but can also be a contribution to research for the lean construction community. In any lean implementation, spreading an idea or a program throughout a large organization is a challenge, especially in a company were top-down orders are not an option, instead the workshop aim to inspire and motivate teams so they want to achieve quality results. In this case, we worked to increase the practice of understanding and aligning expectations with all stakeholders, which is central to DPR's BBQ quality approach and for DPR's quality framework, based on Build with Passion, Clarity and Knowledge.

Several papers have studied teaching lean practices, such as lean leadership training (Hackler et al. 2018), teaching choosing by advantages (Arroyo et al. 2019), teaching lean construction (Tsao et al. 2013 and Nofera et al. 2015). However, due to the novelty of the BBQ approach, no publications on how to train people on the topic have been developed. This paper closes that gap. The paper describes the experience of designing a companywide virtual workshop to shift the quality mindset from build and check it to proactively align expectations to avoid rework, and describes the findings of having conducted the training with multiple teams. The paper also discusses the struggles, changes, and results to get more engagement and implementations. Finally, we discuss struggles and present recommendations for escalating the BQB workshop.

\section{METHODOLOGY}

The methodology to develop and test the workshop followed Design Science Research (Hevner, 2007), where the artifact was the BQB workshop. DSR aims to test an Applied Science/Engineering (AS/E) to produce a scientific methodology (artifact) for construction projects, which are phenomena that vary according to time, contexts, and application conditions (Hevner, 2007). Design Science Research (DSR) is useful to evaluate evidence of learning and gain knowledge to inform best practices (Van Aken 2004). 
In order to test the success of the BQB workshop several measures were used, such as 1) the number of participants that graduated from the program and its distribution by role and by region, 2) the commitment to implement DPR's BBQ from the participants and actual implementation verified on a follow up session, and 3) the participant's postevaluation of the workshop through a survey providing feedback on the workshop's, content, format, and impact.

\section{BQB DEVELOPMENT AND ADJUSTMENTS}

The authors of this paper developed the first Building Quality Builders (BQB) pilot workshop with the support of DPR's Learning and Development team. This workshop was inspired by the company's BBQ approach. The pilot included 8 sessions, 1 session per week, with topics ranging from why a quality approach focused on behavior was needed to the leadership's role in implementing this approach and the relevance of language in quality. Figure 1 shows the evolution of the first 6 rounds (each round represents one group that took the workshop).

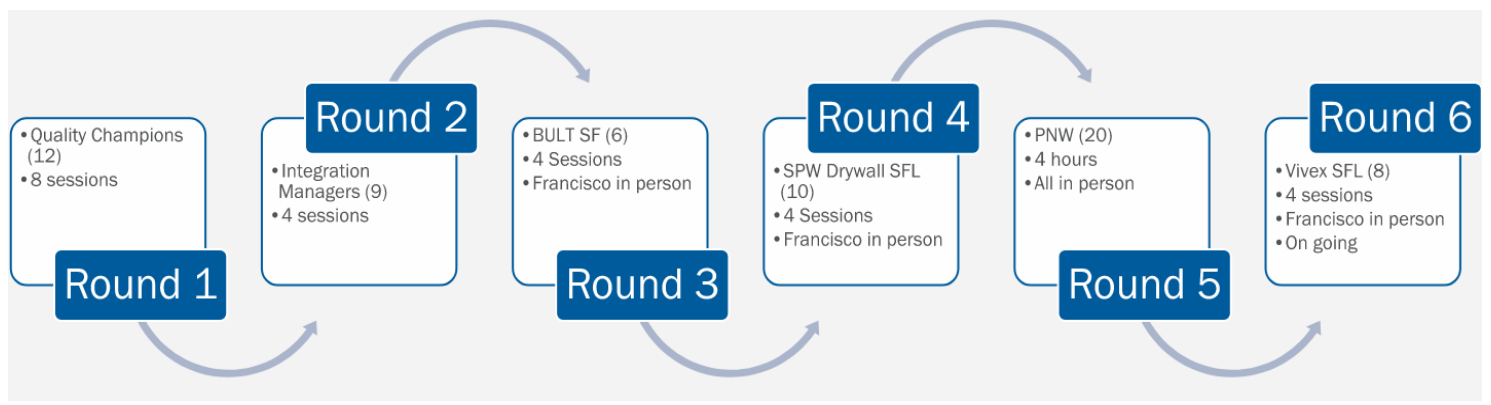

Figure 1: BQB Format Variation Changes

The pilot of BQB followed the flipped classroom approach, where all the learning material is available for participants before every meeting with the facilitator. The workshop material consisted of short videos, mostly DPR's internal videos of discussions held on a quality summit and a few project implementation stories, reading materials (Including Spencley et al. 2018), and live discussions with the facilitator(s) and supplemental resources. BQB pilot session was facilitated by the first author and the second author played the role of a participant along with other people. After each round the BQB workshop was adjusted based on the post-evaluation survey, and plus deltas given during the workshops.

Challenges found in launching and conducting the pilot, and the actions taken to address these challenges are:

- Commitment to an 8-week training was difficult for some participants, especially for the ones working on project sites whose schedule was highly variable. This helped to change the 8-week program to a 4-week program keeping the flipped classroom approach.

- Ideas and theory needed to be translated into more tangible examples. This helped to insert examples of projects that have implemented certain pieces of the BBQ process, how they did it, and what they achieved through this so that the workshop participants can visualize the implementation of the process in real cases.

- Some reading materials were too theoretical or extensive for some of the participants. This helped realized the struggle of people in projects to keep up with 
readings and we replace these for videos that included similar messages that the readings intended to deliver.

- Some videos were too long. With the support of Learning and Development team some videos were edited aiming for shorter times so participants can watch them in small batches to accommodate their schedule.

Round 2 of BQB was tested with Integration Managers, who are in charge of supporting project teams set up best in class practices and help them choose the right tools. Some of the challenges highlighted after this round were:

- How to to escalate best in class practices across a variety of projects, including small renovations and large projects. Even when some project stories were added, the feedback was to develop more implementation examples from a variety of project types, especially for small projects.

- More clarity was needed on where was appropriate to identify DF, when to start implementing, and how to engage with external stakeholders. This led to looking for more case studies and develop more videos.

In parallel with $\mathrm{BQB}$ training, the authors kept learning from project implementation stories. Gomez et al. (2019) described a case study where the concept of BBQ was applied to the delivery of the component of architectural shear walls for a large project. This implementation highlighted areas for improvement in the delivery of quality components such as the need to:

- Make quality a responsibility of every individual and not just the project's quality champion or quality manager.

- Increase the awareness of DPR's quality approach focus on behaviors.

- Highlight best builders' behaviors by providing real case examples where BBQ was implemented.

- Create a quality implementation plan at the project level that engages every participant who has a stake in the delivery of specific quality components or services.

Round 3 was tested with a Business Unit Team that lead operations in South Florida. The team gave the following feedback:

- The team valued the materials, but the workshop had to be more action-oriented. This led to rethink all the prework questions to motivate participants to think about how they will implement BBQ on their projects or groups and to arrange all materials so the last session finished with an action plan.

- Better management of when this information is presented to teams. This lead to identify the project on the SE where new rounds will be tested, aiming for the early stages of the projects.

- Some videos were still long, and some had audio issues, specially wen it was a presentation recorded in a computer. This led to hire a videographer and make more professional videos capturing project stories.

Round 4 was tested on a self-perform team focused on Drywall. For this round, some shorter videos on drywall were added and most reading materials were only provided as additional materials, but not mandatory pre-work. The feedback was that the workshop was very helpful, but some members would have preferred an in-person session. 
Round 5 was compressed and develop in-person in 4 hours, some videos were sent as pre-work, but not all participants. Local quality champions presented stories and some role-play exercises were developed. The feedback of the session was positive; however, it was not possible to use all the materials. The conclusion was that keeping the flip classroom approach and the 4-week meetings was more valuable for participants.

Round 6 was presented to a project team in the early stages, one of the facilitators was on site and the other connected remotely. The team was excited about implementing this approach and the timing of the content was appropriate. Also, in round 6 we added a follow-up session 1 month after the last session to check on the team implementation.

From rounds 7 to 26 the format was the same as in round 6. We keep including new videos as they were developed for the pre-work, the new videos focused on interviewing teams and telling their perspectives on implementation, some project teams also included owners on the video stories. In addition, some of the sessions were offered to anyone in a region regardless of role, this helped tested the content on people working on small projects where having the whole team in the training at the same time was not practical. Also coordinating the right timing for teams has proven to be challenging. So, we offered a session for teams and open to anyone on a Business Unit or region.

\section{Introducing Psychological SAFety into Conversations about QUALITY}

The construct of psychological safety has been linked to teams' learning behaviors and better team performance in multiple industries ranging from manufacturing to product development (Edmondson 2012, Edmondson 2018). In construction, as in other industries, learning is fundamental to keep improving and mitigating or eliminating issues that impact the quality, safety, and overall delivery of value (evidencing by rework, accidents, delays, cost overruns, and loss of trust). Gomez et al. (2020) introduced arguments that link psychological safety with the specific impact it can have on quality in construction projects. In summary, psychological safety is needed to raise questions or concerns about quality, and to lead conversations for aligning expectations with several Stakeholders.

BQB rounds 19 and 20 were used to introduced psychological safety into the conversations surrounding the delivery of quality components. This introduction to psychological safety included four steps: 1) presenting the definition of psychological safety, 2) highlighting its role in delivering quality, 3) discussing how psychologically safe participants feel on project teams with different project stakeholders, and 4) conducting an on-hands exercise where participants can observe and experience aspects of psychological safety.

The first and second steps of introducing psychological safety into the workshop consisted on conversations where participants described first their understanding of psychological safety and then the facilitators introduced the concept to the group together and discussed its relevance for delivering quality components. The third step aimed to grasp an overview of how psychologically safe participants feel when they work in construction projects and interact with multiple stakeholder groups, particularly with people from their own company itself (i.e., general contractor employees), owners, architects, and other subcontractors/trade partners. Figure shows an example of the BQB 20 participants' responses to the question "how psychologically safe do you feel with these stakeholder groups?" The last and fourth step consisted of an exercise where participants were put in a certain scenario where they are asked to say "No" when they 
receive a request. In the scenarios given for the exercise, participants are paired with another participant whose role can be seen as being a position of a higher or lesser power (i.e., an owner or a subcontractor respectively).

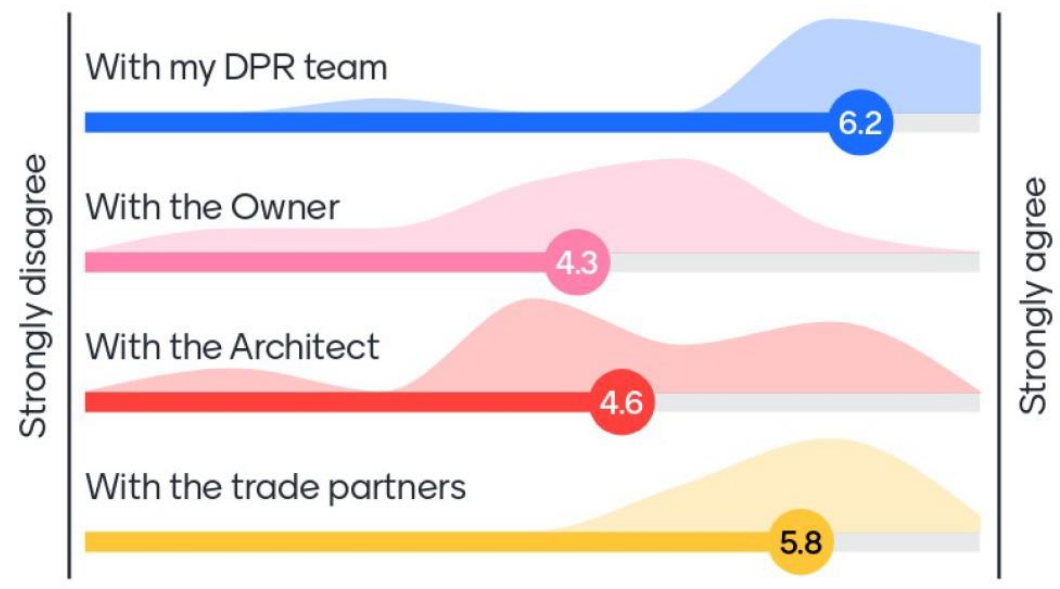

Figure 2: Participants Feeling of Psychological Safety with Different Stakeholders Groups from the Perspective of the General Contractor in BQB Round 20

\section{EXERCISE ON ASSERTIONS (FACTS) AND ASSESSMENTS (STORIES)}

Gomez (2020) described the importance of the Language Action Perspective (LAP) theory in delivering quality components. LAP describes the different speech acts that can be part of a conversation. Among those speech acts, the assertions (also known as facts) and assessments (also known as stories) are fundamental for avoiding misunderstanding and confusion around quality expectations.

Round 19 and 20 also introduced an exercise where participants were asked to describe a picture of an architectural shear wall and a stairs mock-up. In their descriptions, they were challenged to differentiate whether what they have included in their list to describe the component was indeed an assertion (a fact that cannot be neglected e.g., the measure of the wall provided in the picture), or an assessment (an assessment that can be subject to interpretation). Round 23- 26 we kept the exercise on identifying facts and stories using the wall and stair examples.

\section{BQB CURRENT ForMaT}

The current format of BQB is based on a 4-week course plus a follow-up session a month after the latest session. BQB is facilitated internally by DPR Quality Leaders.

BQB continues to follow the Flipped Classroom approach. The course consists of short videos of DPR teams presenting their implementation stories now using a variety of project types, including small and large projects, different core markets, and also different perspectives based on roles. The class now also provides a summary of quality tools (A3 templates for DF, QIP templates, etc.). The applied learning activities described above (i.e., exercise on facts and stories) are also part of the live discussions with the facilitator(s). Participants are asked to dedicate 2 hours per week, totaling around 8 hours across 4 consecutive weeks, consisting of 1-hour of pre-work (watching short videos, reading short documents, and answering 5 questions) and 1-hour team call where participants engage in a safe and productive conversation. In addition, participants are asked to go to a 1-hour follow-up session that allows everyone to share what has been working and what needs to change.

The BQB workshop current agenda includes: 
- Session 1 -Why a Behavioral Approach to Quality?

- Session 2 -Quality Language and Leadership

- Session 3 -How to Apply the DPR Quality Approach? (videos and materials include pursuit, pre-construction, construction, and post-construction examples)

- Session 4 -Action Plan

- Follow up - 4 weeks after session 4 .

\section{RESULTS}

This section presents the results from the workshop in terms of feedback received from participants and examples of implementation where re-work was avoided.

\section{PARTICIPANTS}

Over the 26 BQB rounds, 253 participants have graduated. Participants have been mostly joining from the North West (NW) region, which includes DPR offices in San Francisco, Redwood City, Sacramento, San Jose, and Seattle. This is mainly due to stronger leadership support from the NW region. Other regions where participants have voluntarily enrolled in the training are South West (SW), South East (SE), North East (NE), Central, Europe, and India (Figure 3).

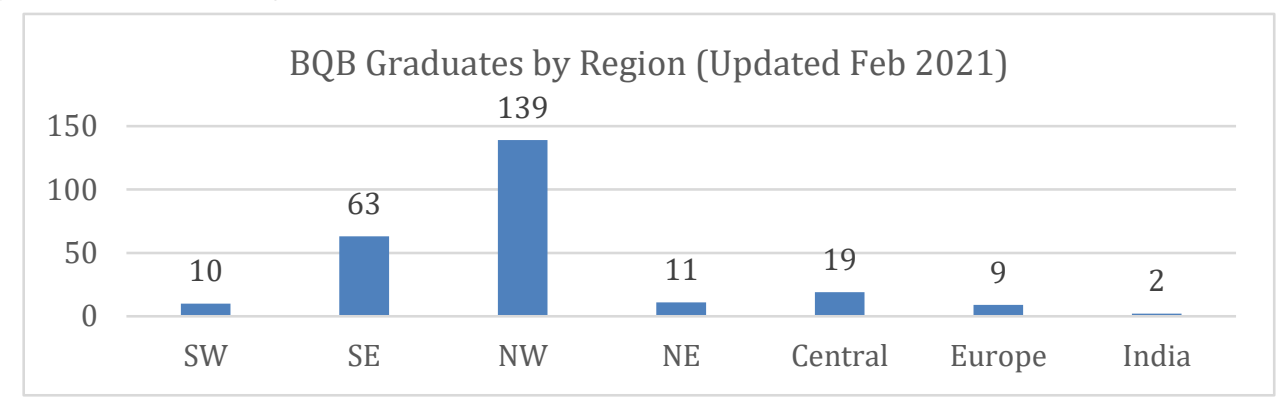

Figure 3: Number of BQB Participants by Region

Figure 4 shows BQB Participants by role. A variety of roles have participated in the training, with superintendents being the biggest group, followed by PMs and PEs.

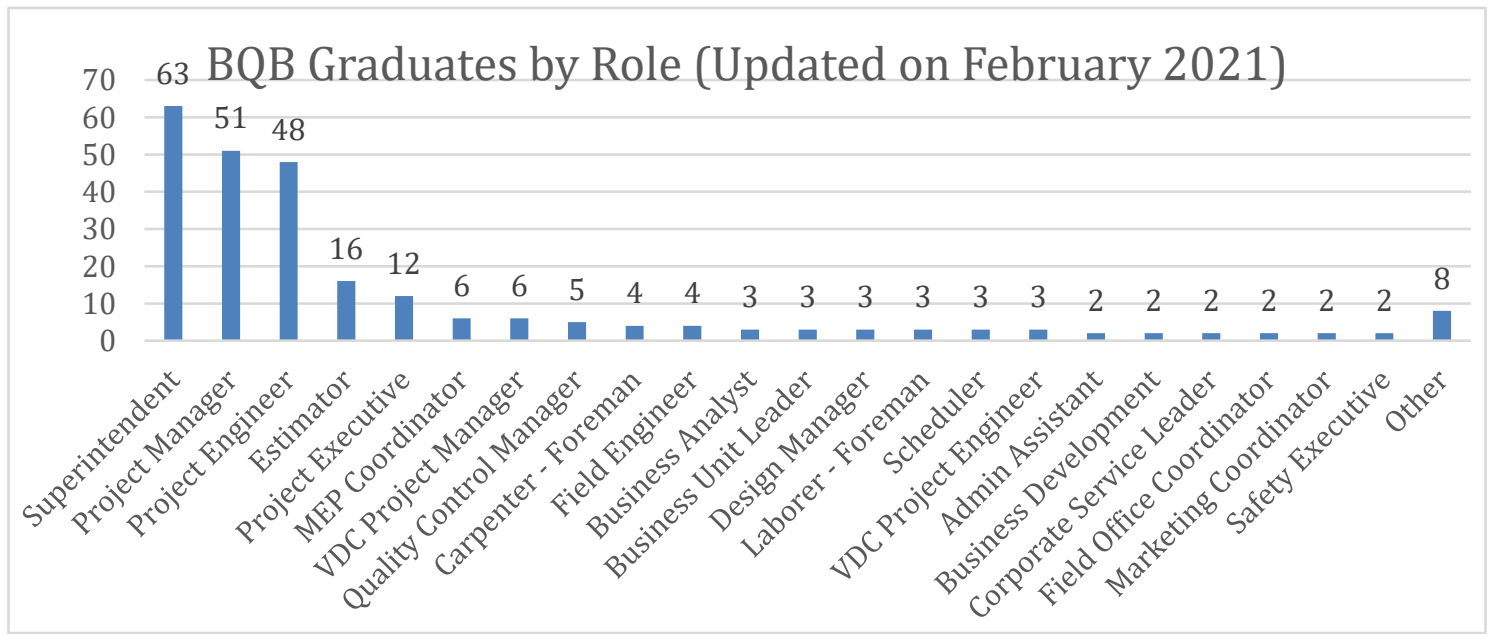

Figure 4: Number of BQB Participants by Role 


\section{COMmitMent to ACTiON}

In the last session of the training, participants are asked to develop an action plan in which they explain what they plan to do to implement what they have learned through the training. Table 1 shows an example of commitments made on BQB round 24 for a project team in the NE building a hospital project. A follow-up session was scheduled 1 month after the last BQB session, participants performed all committed actions.

Table 1: BQB Group 24 Strategic Action Items

\begin{tabular}{|c|c|c|}
\hline & Strategic Action & Responsible \\
\hline 1 & Create DFOW log & Project Manager (PM) \\
\hline 2 & Creating Visuals for Stairs & Project Engineer) \& PM \\
\hline 3 & $\begin{array}{l}\text { Taking the visuals and making sure they are part of Foreman } \\
\text { meetings and post them on the field in the areas. }\end{array}$ & Superintendent \\
\hline 4 & $\begin{array}{l}\text { Provide support in the process, be engaged in quality conversations. } \\
\text { Provide input on problem areas. }\end{array}$ & Precon \\
\hline 5 & $\begin{array}{l}\text { Ensure Quality approach gets implemented in next projects. GMP. } \\
\text { Planting seed in proposals. }\end{array}$ & Project Executive \\
\hline
\end{tabular}

An A3 visual was developed for the Distinguishing Features of the stairs (Figure 5). The team collaborated with the project architect and owner to dive deeper into the construction details and found out some items were not clear, so they developed alignment and described MAC for them. The team was happy that all these details were sorted beforehand and agreed with the architect and owner, so all rework is avoided on this scope of work.
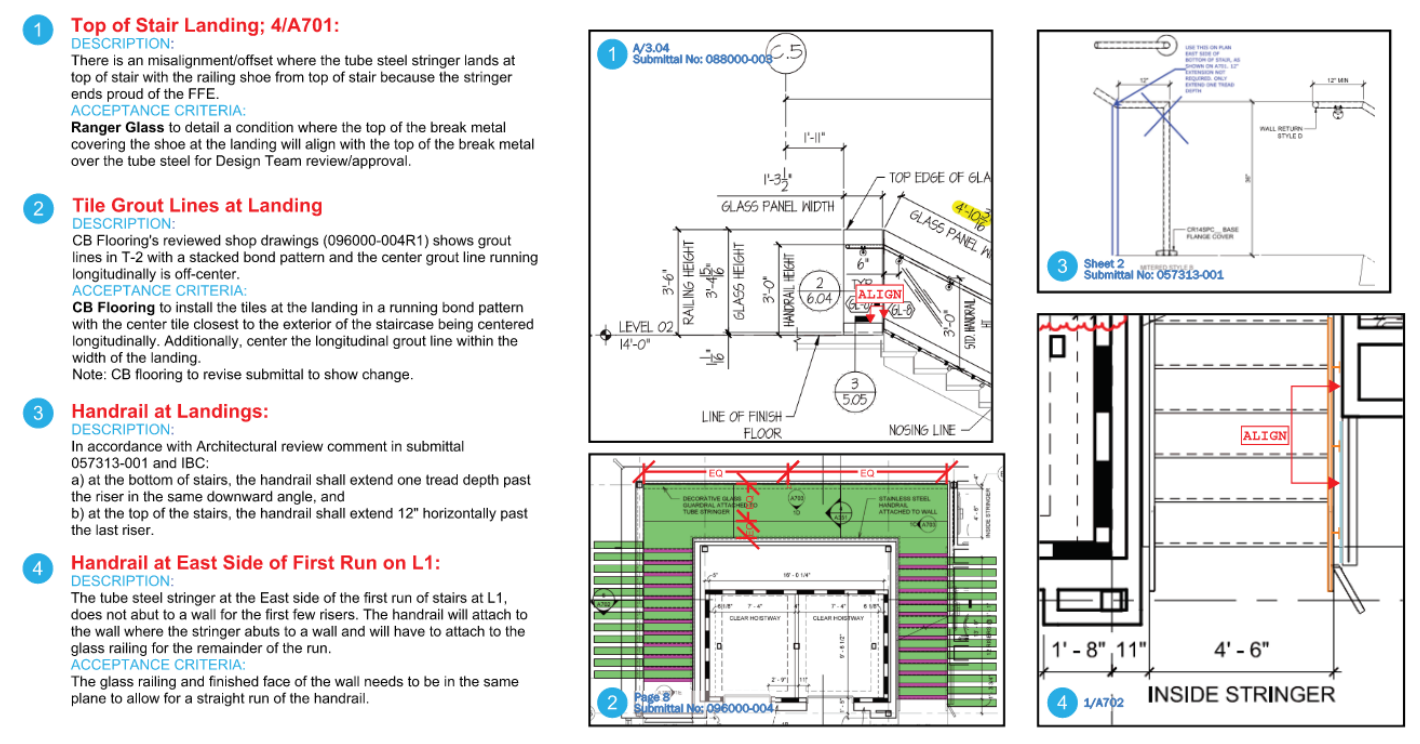

Figure 5: Example of Visual explanation of Distinguishing Features for a Stair.

\section{Participants Feedback}

Feedback from participants was collected in a voluntary post-evaluation survey (48 respondents out of 253 participants). This section summarizes the results of BQB groups from 1 to 20. Participants were asked whether they would recommend the training to others at the company. Results show that $98 \%$ (47 out of 48) of the participants would recommend this program to their co-workers. When participants were asked who should 
go through the training the answers point out to PEs, Superintendents, PMs, PXs, Preconstruction, and BULT, many said everyone in the company.

In addition, participants' testimonials emphasized the benefit of the training and how it would change their behaviors moving forward when asked about their takeaways and future actions:

"The class significantly changed my view of quality and how awesome no rework can be, by being on the top of our game. I completely believe that anyone executing work should go through this training" - DPR Superintendent

"I am going to ask more questions, earlier to ensure that the "Unknown" is turned into the "Known" - Integration Manager

"The class reinforced to me the reality that we are a service industry. Most of our competition can build. We set ourselves apart when we have a process for capturing what's important to our clients and we deliver consistently. I think DFOW is a game-changer." - SPW Drywall Team Member

"When engaging the client, I will continue the conversation of what is important to them and how we incorporate quality." - Business Development "DFOW aren't just applicable to the finished product but processes. Maintaining some type of consistency office-wide can help reinforce making our quality program and DFOW a habit." - Project Engineer

"It (Quality) represents an opportunity for DPR to drive higher Gross Margins. We need to create a common language that gives context to Quality as a value position." - Business Unit Leader Team Member

\section{CONCLUSIONS}

This paper described the lessons learned through the development and teaching of BQB, a virtual workshop for training employees working in the construction industry on the BBQ approach towards quality. Following the DSR method, the workshop has followed a continuous improvement cycle of testing and refinement. The paper explains the different changes made to the pre-work, format to deliver the content, and discussion exercises. These changes were progressively made considering feedback from the postevaluation survey that participant gave regarding the format and content of the workshop.

Each addition to the workshop responded to specific needs. For example, the addition to include discussions around psychological safety into the workshop aimed to cover the gap of helping participants in the workshop understand how psychological safety can impact their work of delivering quality. Similarly, the addition to include an exercise on language action perspective basics to differentiate assessment versus assertions aimed to increase clarity on the way workshop participants express their expectations and understand other people's expectations properly. Another example is the addition to focus on getting commitment to action. Participants were asked to develop an action plan for how they could implement what was learned in the workshop sessions to the work they do.

Participants who took the workshop highly recommend taking this workshop to their peers, business unit leaders, and some recommended everyone in the company taking it. Their testimonials showed different areas where they observed the workshop being helpful to them in meeting quality expectations. The BQB workshop has helped 
participants understand ways for how to better deliver quality in any time of work they do.

\section{ACKNOWLEDGMENTS}

We would like to acknowledge the contribution of several DPR employees that have contributed with their ideas to design elements of the workshop and to facilitate it, including Ralph Romayor, Francisco Reyna, JD Nelson, and Erica Sharp. In addition, we would like to acknowledge the role of Learning and Development teammates at DPR that have provided state of the art platforms and support in material development, especially thanks to Tina Chong for her continued support. Finally would like to acknowledge Hooman Shahrokhi for obtaining permission to share an A3 with a DF example.

\section{REFERENCES}

Arroyo, P., Christensen, R., Schöttle, A. \& Long, D. (2019). "Lessons Learned on Teaching Choosing by Advantages" In:, Proc. 27th Annual Conference of the International Group for Lean Construction (IGLC). Dublin, Ireland, 3-5 Jul 2019. pp 405-416.

Van Aken, J.E. (2004). "Management Research Based on the Paradigm of the Design Sciences: The Quest for Field-Tested and Grounded Technological Rules." Journal of Management Studies, 41(2), 219-246.

Gomez, S. , Huynh, R. , Arroyo, P. , Ballard, G. , Tommelein, I. \& Tillmann, P. (2019). "Changing Behaviors Upstream to Achieve Expected Outcomes" In:, Proc. 27th Annual Conference of the International Group for Lean Construction (IGLC). Dublin, Ireland, 3-5 Jul 2019. pp 13-24.

Gomez, S. , Ballard, G. , Arroyo, P. , Hackler, C. , Spencley, R. \& Tommelein, I. D. (2020), "Lean, Psychological Safety, and Behavior-Based Quality: A Focus on People and Value Delivery" In:, Proc. 28th Annual Conference of the International Group for Lean Construction (IGLC). Berkeley, California, USA, 6-10 Jul 2020. pp 97-108

Edmondson, A.C. (2012). Teaming: How Organizations Learn, Innovate, and Compete in the Knowledge Economy. John Wiley \& Sons

Spencley, R. , Pfeffer, G. , Gordon, E. , Hain, F. , Reed, D. \& Marosszeky, M. (2018) "Behavior-Based Quality, Case Study of Closing the Knowing-Doing Gap" In:, 26th Annual Conference of the International Group for Lean Construction. Chennai, India, 18-20 Jul 2018. pp 1170-1181.

Hackler, C. , Byse, E. , Alves, T. C. L. \& Reed, D. (2018), “Lean Leadership Training: Lessons From a Learner-Centered Analysis" In:, 26th Annual Conference of the International Group for Lean Construction. Chennai, India, 18-20 Jul 2018. pp 484494.

Tsao, C. C. , Azambuja, M. , Hamzeh, F. R., Menches, C. \& Rybkowski, Z. K. (2013). "Teaching Lean Construction Perspectives on Theory and Practice" In:, Formoso, C. T. \& Tzortzopoulos, P., 21th Annual Conference of the International Group for Lean Construction. Fortaleza, Brazil, 31-2 Aug 2013. pp 977-986.

Nofera, W. , Abdelhamid, T. S. \& Lahouti, A. (2015), "Teaching Lean Construction for University Student(S)” In:, Seppänen, O., González, V. A. \& Arroyo, P., 23rd Annual Conference of the International Group for Lean Construction. Perth, Australia, 29-31 Jul 2015. pp 424-433. 